Research Paper

\title{
Adenoviral and Adeno-Associated Viral Vectors- Mediated Neuronal Gene Transfer to Cardiovascular Control Regions of the Rat Brain
}

Yanling Zhang ${ }^{1, *}$, Yongxin Gao ${ }^{2, *}$, Robert C. Speth ${ }^{2,3}$, Nan Jiang ${ }^{2}$, Yingying Mao ${ }^{1}$, Colin Sumners ${ }^{2}$ and Hongwei $\mathrm{Li}^{1}{ }^{1}$

1. School of Biotechnology, Southern Medical University, Guangzhou, China;

2. Department of Physiology and Functional Genomics and McKnight Brain Institute, University of Florida, Gainesville, Florida, USA;

3. Department of Pharmaceutical Sciences, College of Pharmacy, Nova Southeastern University, Fort Lauderdale, FL, USA.

* Both authors contributed equally to this work.

$\triangle$ Corresponding author: Hongwei Li, School of Biotechnology, Southern Medical University, 1023 South Shatai Road, Guangzhou, Guangdong 510515, China. Phone: 86-20-61648555; Fax: 86-20-61648555. E-mail: hongwei1@yahoo.com.

(c) Ivyspring International Publisher. This is an open-access article distributed under the terms of the Creative Commons License (http://creativecommons.org/ licenses/by-nc-nd/3.0/). Reproduction is permitted for personal, noncommercial use, provided that the article is in whole, unmodified, and properly cited.

Received: 2012.12.12; Accepted: 2013.03.1I; Published: 2013.03.20

\begin{abstract}
Viral vectors have been utilized extensively to introduce genetic material into the central nervous system. In order to investigate gene functions in cardiovascular control regions of rat brain, we applied WPRE (woodchuck hepatitis virus post-transcriptional regulatory element) enhanced-adenoviral (Ad) and adeno-assoicated virus (AAV) type 2 vectors to mediate neuronal gene delivery to the paraventricular nucleus of the hypothalamus, the nucleus tractus solitarius and the rostral ventrolateral medulla, three important cardiovascular control regions known to express renin-angiotensin system (RAS) genes. Ad or AAV2 harboring an enhanced green fluorescent protein (EGFP) reporter gene or the angiotensin type 2 receptor gene were microinjected into these brain regions in adult rats. Our results demonstrated that both AAV2 and Ad vectors elicited long-term neuronal transduction in these regions. Interestingly, we found that the WPRE caused expression of GFP driven by the synapsin I promoter in pure glial cultures or co-cultures of neurons and glia derived from rat hypothalamus and brainstem. However, in rat paraventricular nucleus WPRE did not cause expression of GFP in glia. This demonstrates the potential use of these vectors in studies of physiological functions of certain genes in the cardiovascular control regions of the brain.
\end{abstract}

Key words: adenoviral vector; adeno-associated viral vector; cardiovascular control regions of brain; neuron; gene transfer.

\section{Introduction}

The systemic RAS plays a critical role in cardiovascular homeostasis. All components of the RAS are also known to be produced cell-specifically within specific brain regions, although the role of the brain RAS relative to the systemic RAS has remained a puzzle due to the difficulty of differentiating these two systems. Powerful new experimental strategies such as somatic gene transfer via recombinant viral vectors offer potential avenues for analyzing these important systems independently[1].

Attenuated viral vectors, such as adenovirus, lentivirus, herpes simplex virus, and adeno-associated virus have been used to introduce genetic material into the nervous system[2,3]. Adenovirus vectors 
permit the introduction of relatively large DNA sequences, are easy to construct and propagate, are safe to use, and can be cultivated to high titers. However, even though the brain is immune privileged compared with other organs, it has been shown that an inflammatory response is generated at commonly used viral doses ( $10^{7}$ infectious units or more) given via the intracerebral route. In addition, adenoviral-mediated transgene expression in the brain may be relatively short lived due to elimination of the virus by the immune response[4,5]. We have previously shown that the human synapsin 1 (SYN) gene promoter coupled with an adenoviral vector produces neuron-specific transgene expression in primary neuronal cultures from rat hypothalamus and in adult rat paraventricular nucleus (PVN), but the over-expression diminished relatively quickly in vivo[6]. It has been suggested that, by using strong promoters, fewer immunogenic viral particles would be needed to mediate transgene expression and hence the inflammatory response could be reduced. To achieve this enhanced expression, we considered the use of post-transcriptional enhancer elements in conjunction with cell-specific promoters. The woodchuck hepatitis virus post-transcriptional regulatory element (WPRE) is a powerful viral enhancer element that is thought to improve expression by modification of RNA polyadenylation, RNA export, and/or RNA translation[7]. Therefore, we incorporated WPRE with a human SYN promoter-enhanced green fluorescent protein (EGFP) reporter cassette in the construct Ad5-SYN-EGFP-WPRE. Next, we assessed the influence of WPRE on SYN promoter-mediated foreign transgene expression in cortical neuronal cultures and the PVN.

Recombinant adeno-associated virus (rAAV) has been utilized extensively in the nervous system as a gene delivery vector. It targets primarily neurons in the nervous system and results in sustained long-term expression of transgenes[8-11]. In this study, we used an AAV2 vector to produce longer-term (at least 2 months) neuronal overexpression of foreign genes (EGFP or the angiotensin type 2 receptor [AT2R]) in the PVN and other cardiovascular control regions of the brain, such the nucleus tractus solitarius (NTS) and rostral ventrolateral medulla (RVLM).

\section{Materials and Methods}

\section{Animals}

In this study we used a total of 18 male Sprague-Dawley (SD) rats (9-10 weeks old), purchased from Charles River Farms (Wilmington, MA). The University of Florida Animal Use and Care Commit- tee approved all animal procedures.

\section{Preparation of Viral Vectors}

Preparation of the adenoviral vectors Ad5-SYN-EGFP and Ad5-SYN-EGFP-WPRE (Figure 1A) was performed exactly as detailed previously[12]. The viral titers were $1.4 \times 10^{10}$ infectious units (ifu) $/ \mathrm{ml}$ for Ad5-SYN-EGFP and $2.5 \times 10^{10} \mathrm{ifu} / \mathrm{ml}$ for Ad5-SYN-EGFP-WPRE. Expression of EGFP was driven by a human SYN promoter. The WPRE, which enhanced the expression of transgenes, was present downstream of EGFP.

Two AAV vectors, AAV2-CBA-AT2R-WPRE and AAV2-CBA-EGFP-WPRE, were constructed for this study (Figure 1B). Both of these constructs contained expression cassettes flanked by rAAV2 terminal repeats. Expression of EGFP and the angiotensin type 2 receptor (AT2R) was driven by a chicken beta actin promoter (CBA) with human cytomegalovirus (CMV) enhancer. Vectors were propagated in HEK293 cells using pDG as the helper plasmid and purified with a single-step gravity-flow column, and the purity of viral preparations was assessed by SDS-polyacrylamide gel electrophoresis [13]. Copies of viral genome DNA were determined by quantitative PCR [14]. The vector doses were expressed as genome copies $(\mathrm{gc})$ and the titers were $1.0 \times 10^{12}$ $\mathrm{gc} / \mathrm{ml}$ for AAV2-CBA-EGFP-WPRE and $5.0 \times 10^{11}$ $\mathrm{gc} / \mathrm{ml}$ for AAV2-CBA-AT2R-WPRE.

\section{Preparation of neuronal and astroglial cultures viral transduction}

Primary neuronal cultures, and pure astroglial cultures were prepared in 24-well plates or $35 \mathrm{~mm}$ dishes from the hypothalami and brain stems or from the cerebral cortex of newborn SD rats, as detailed previously[15]. Cells were transduced with viral vectors at day 10 after preparation and 5 days later the expression of foreign genes was analyzed.

\section{Viral injections into PVN, NTS and RVLM}

Male SD rats were anesthetized with a mixture of $\mathrm{O}_{2}(1 \mathrm{~L} / \mathrm{min})$ and $4 \%$ isoflurane, and placed in a Kopf stereotaxic frame. Anesthesia was maintained using an $\mathrm{O}_{2}$ /isoflurane $(2 \%)$ mixture delivered through a specialized nose cone for the duration of the surgery. Microinjections of the viral vectors (Ad-SYN-EGFP-WPRE, $1 \mu \mathrm{l}$ of $5 \times 10^{6}$ ifu; AAV2-CBA-EGFP-WPRE, $1 \mu \mathrm{l}$ of $1.0 \times 10^{9} \mathrm{gc}$; AAV2-CBA-AT2R-WPRE, $1 \mu \mathrm{l}$ of $5.0 \times 10^{8} \mathrm{gc}$ ) were performed as previously described [5], using the following stereotaxic coordinates. PVN (bilateral injections): $1.9 \mathrm{~mm}$ posterior to bregma, $\pm 0.2 \mathrm{~mm}$ lateral to the midline, and $7.8 \mathrm{~mm}$ ventral to the skull surface; 
RVLM (bilateral injections): $12.36 \mathrm{~mm}$ posterior to bregma, $\pm 2.0 \mathrm{ML}, 9.7 \mathrm{~mm}$ ventral to the skull surface; NTS: $1.5 \mathrm{~mm}$ lateral to midline, $1.0 \mathrm{~mm}$ rostral to obex, and $1.5 \mathrm{~mm}$ below the dorsal medullary surface. At least 4 SD rats were used for injection of the individual viral vectors at each of these sites.

\section{Real-time RT-PCR for the detection of EGFP mRNA}

Total RNA was prepared from the transduced cultures or tissues using an RNeasy kit (Qiagen, Valencia, CA). EGFP mRNA was analyzed via quantitative real time RT-PCR as detailed by us previously[16].

\section{Immunocytochemical Procedures}

Neuronal cultures: Viral vector-treated cells were washed briefly with Dulbecco's phosphate-buffered saline (PBS) and then fixed for $10 \mathrm{~min}$ with PBS containing $0.1 \%$ Tween 20 (PBS/Tween) and $4 \%$ formaldehyde solution. The fixed cells were permeablized in PBS containing $0.3 \%$ Triton X-100 (Sigma, 9002-93-1) for $20 \mathrm{~min}$. Immunocytochemistry was then performed on the fixed cells as detailed previously[17], using a neuron-specific primary antibody (monoclonal anti-NeuN antibody, 1:1000; Chemicon International, Temecula, CA) or an astrocyte-specific monoclonal anti-glial fibrillary acidic protein [GFAP] (1:1000; Chemicon)), followed by Alexa Fluor 594 goat anti-mouse IgG (1:1000; Molecular Probes, Eugene, OR) as the secondary antibody. NeuN and GFAP immunoreactivity (red) and green fluorescence (from GFP) were detected using an Olympus BX41 fluorescence microscope.

Brain Slices: Viral vector-injected rats were anesthetized with isoflurane and perfused transcardially with $100 \mathrm{~mL}$ of $0.9 \% \mathrm{NaCl}$ followed by $100 \mathrm{~mL}$ of $4 \%$ paraformaldehyde in PBS. The brain was removed, post-fixed in $4 \%$ paraformaldehyde solution for 2-3 hours, and transferred to a phosphate buffer containing $20 \%$ sucrose. Following this, the brain tissues containing the PVN, RVLM, or NTS were sectioned in the coronal plane $(30 \mu \mathrm{m})$ using a freezing microtome. Sections were floated onto glass slides ready for immunostaining. Antibodies were monoclonal anti-NeuN primary antibody $(1: 100)$ and Alexa Fluor 594 goat anti-mouse IgG (1:1000; Molecular Probes) secondary antibody. NeuN immunofluorescence and green fluorescence (from GFP) were detected using an Olympus BX41 fluorescence microscope

\section{Receptor autoradiography}

At 2 months post AAV-CBA-AT2R injection, rats were deeply anesthetized with sodium pentobarbital and were perfused transcardially with chilled phosphate buffered saline. The brains were removed quickly, wrapped in aluminum foil, and frozen at -80 ${ }^{\circ} \mathrm{C}$ until sectioned. Coronal sections $(20-\mu \mathrm{m}$ thickness) were cut from rat brains using a cryostat and thaw-mounted onto gelatin-coated slides. The sections were allowed to dry at room temperature and then stored at $-80^{\circ} \mathrm{C}$ until the time of autoradiography. The frozen sections were thawed and in vitro receptor autoradiography was performed using ${ }^{125}$ I-labeled sarcosine $^{1}$, isoleucine ${ }^{8}$ angiotensin II (125I-SI Ang II) as described previously $[18,19]$. The slides were incubated in a solution with $500 \mathrm{pM}{ }^{125 I-S I}$ Ang II and 10 $\mu \mathrm{M}$ losartan (a selective AT1 receptor antagonist) to assess AT2 receptor binding and determine the AT2R expression. Alternate brain sections were incubated with $3 \mu \mathrm{M}$ Ang II to define non-specific ${ }^{125}$ I-SI Ang II binding. The results were analyzed by quantitative densitometry using the computer program MCID ${ }^{\circledR}$ (Imaging Research, St. Catherines, ON). Non-specific binding in the presence of $3 \mu \mathrm{M}$ Ang II was subtracted from "Total binding" in the absence of Ang II to determine specific binding to AT2 receptors.

\section{Results}

\section{Generation and characterization of viral vec- tors}

The construction and characterization of the adenoviral vectors used in this study has been described previously[12]. Ad5-SYN-EGFP and Ad5SYN-EGFP-WPRE are shown diagrammatically in Figure 1A. Unexpectedly, we observed weak SYN promoter-mediated expression during adenoviral passaging in HEK293 cells, a finding also reported by Kugler et al. [20]. The reason for this behavior was not investigated but may be explained by a recent finding that HEK293 cells express many neuron specific proteins, including neurofilament (NF), which suggests this cell line has some neuronal characteristics [21]. In addition, Ad5-SYN-EGFP-WPRE caused stronger GFP fluorescence in transduced HEK 293 cells compared with Ad5-SYN-EGFP (data not shown).

In this study, we also constructed the rAAV2 vectors AAV2-CBA-EGFP-WPRE and AAV2-CBAAT2R-WPRE (Figure 1B). These vectors were purified using an easy-to-do single-step column purification (SSCP) by gravity flow based on affinity to heparin, without ultracentrifugation. Various vector preparations generated by this method have reproducibly showed high titers, infectivity, and purity as previously reported [13]. 
WPRE enhances transgene expression in cortical neuronal cultures transduced with adenoviral vectors

To characterize the effect of the WPRE on SYN promoter activity, we compared EGFP expression in neuronal cultures produced by Ad5-SYN-EGFP and Ad5-SYN-EGFP-WPRE. In transduced primary rat cortical neurons, Ad-SYN-EGFP-WPRE elicited greater green fluorescence in cells from day 1 post-transduction when compared with Ad-SYN-EGFP. At day 3 post-transduction, Ad-SYN-EGFP-WPRE caused a high level of green fluorescence in a large number of cells (Figure 2A), and this fluorescence was closely aligned with neuronal cells as evidenced by immunostaining for the neuron-specific marker NeuN (Figure 2, panels B, C). There was no significant EGFP expression in the few glia that are present in these cultures. Furthermore, real-time RT-PCR analyses revealed that Ad-SYN-EGFP-WPRE produced an $\sim 3$ fold greater increase in EGFP mRNA expression compared with Ad5-SYN-EGFP (Figure 3A).

\section{Ad-SYN-EGFP-WPRE induced EGFP expres- sion in glia from pure glial cell culture or co-cultures}

Residual SYN promoter activity in astrocytes has been reported in astrocyte enriched neuronal cultures[2]. Our results demonstrated that in co-cultures prepared from rat hypothalamus and brain stem, which contain a mix of neurons and glia, strong EGFP expression was observed in both cell types following transfection with Ad-SYN-EGFP-WPRE. Using immunocytochemistry, we demonstrated co-localization of EGFP with immunoreactive GFAP (Figure 2 panels D-F). Furthermore, Ad-SYN-EGFP-WPRE elicited strong EGFP expression in astrocytes contained in glial cell cultures (Figure 2 panel G-I) and produced a 26.6 fold increase of EGFP mRNA compared with Ad-SYN-EGFP without WPRE (Figure 3B).

$1 \mathrm{~A}$

Ad5-SYN-EGFP-WPRE

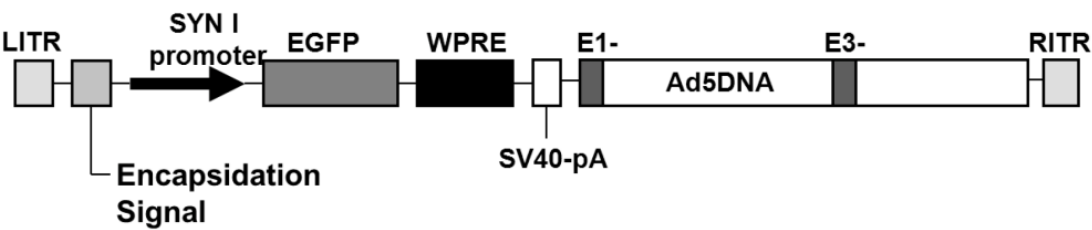

Ad5-SYN-EGFP

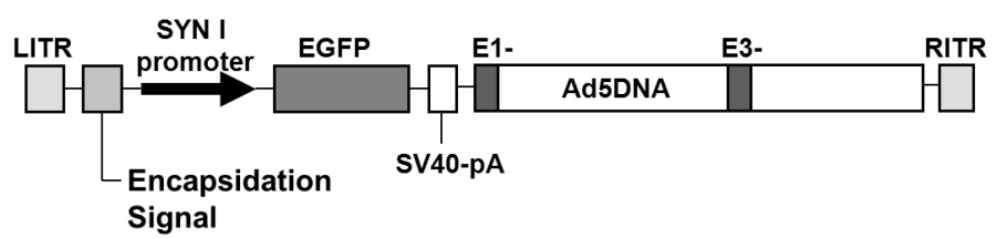

1B

AAV2-CBA-EGFP-WPRE

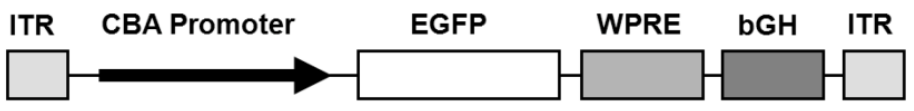

AAV2-CBA-AT2R-WPRE

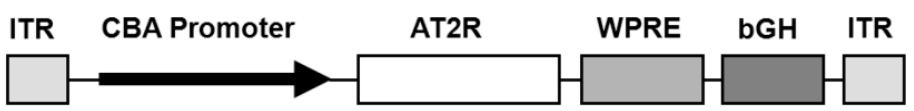

Figure I. Schematic representation of the viral vectors used in this study. (A) Adenoviral vectors, Ad5-SYN-EGFP-WPRE and Ad5-SYN-EGFP. Both adenoviral vectors contain the enhanced green fluorescent protein reporter gene (EGFP). ITR, Ad inverted terminal repeats; SYN I, human synapsin I promoter; WPRE, woodchuck hepatitis virus post-transcriptional control element. (B) AAV2 vectors, AAV2-CBA-EGFP-WPRE and AAV2-CBA-AT2R-WPRE. AT2R, rat angiotensin II type 2 receptor; CBA, chicken beta-actin/CMV hybrid promoter; $b G H$, bovine growth hormone derived polyadenylation site. 

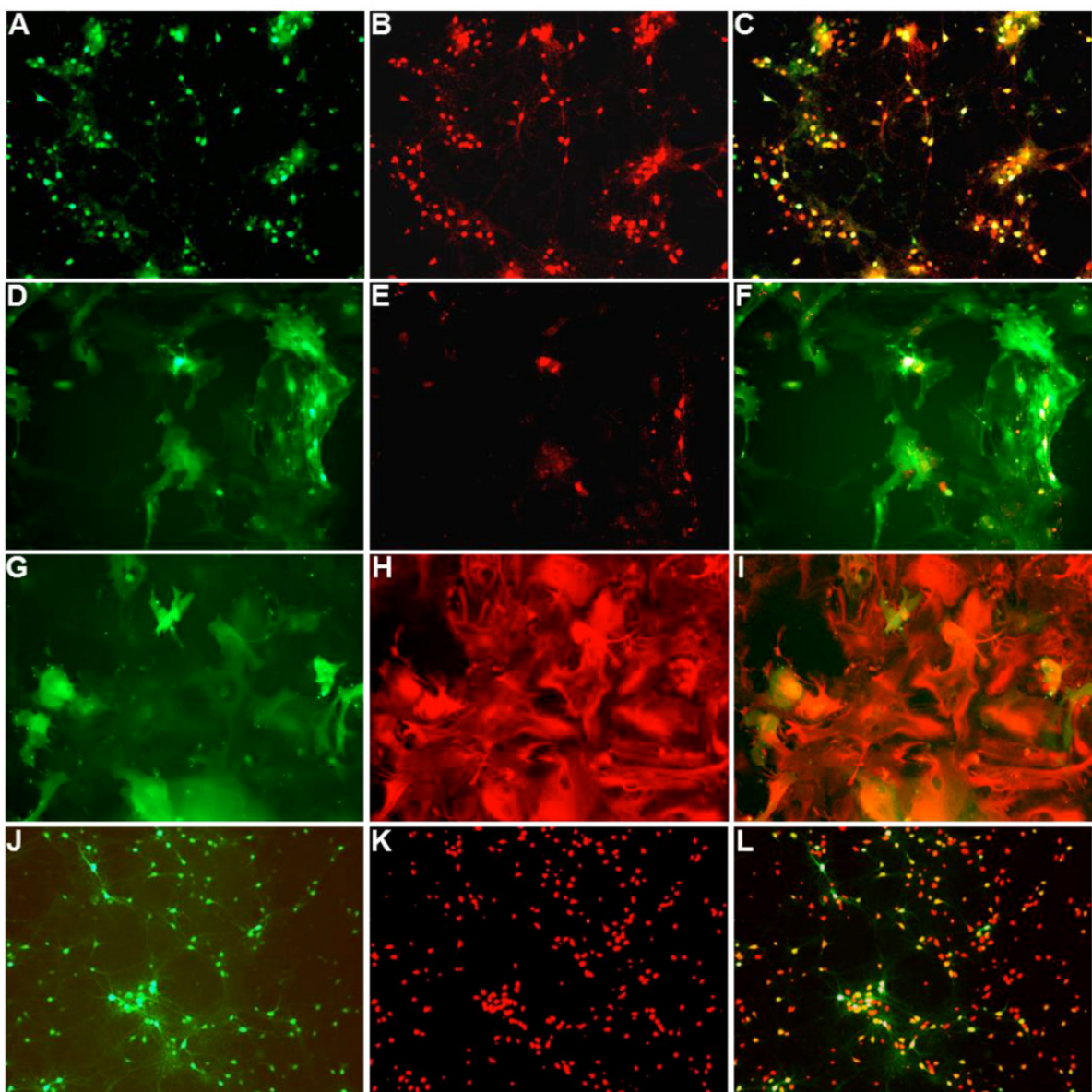

Figure 2. Ad5-SYN-EGFP-WPRE and AAV2-CBA-EGFP-WPRE-mediated transduction of GFP into brain cell cultures.

Primary rat cerebral cortical neurons (panels A-C), primary rat hypothalamus and brain stem co-cultures (panels D-F), and primary glial cell cultures (panels G-I) were transduced with Ad5-SYN-EGFP-WPRE ( $2 \times 10^{7}$ ifu/well) for 3 days as described in the methods. Primary rat cerebral cortical neurons (panels J-L) were incubated with AAV2-CBA-EGFP-WPRE $\left(5 \times 10^{9}\right.$ ifu/well) for 5 days as described in the methods. All incubations were followed by detection of EGFP fluorescence and immunostaining with either anti-NeuN or anti-GFAP antibodies. Panels A, D, G and J are representative images showing EGFP fluorescence. Panels B, E, $\mathbf{K}$ are NeuN immunostaining of the same field of cells in the respective panels $\mathbf{A}, \mathbf{D}$ and $\mathbf{J}$. Panel $\mathbf{H}$ is GFAP immunostaining of the same field of cells in respective panel $\mathbf{G}$. Panels $\mathbf{C}, \mathbf{F}, \mathbf{I}$, and $\mathbf{L}$ are merged images of panels $\mathbf{A} / \mathbf{B}, \mathbf{D} / \mathbf{E}, \mathbf{G} / \mathbf{H}$ and $\mathbf{J} / \mathbf{K}$ respectively.
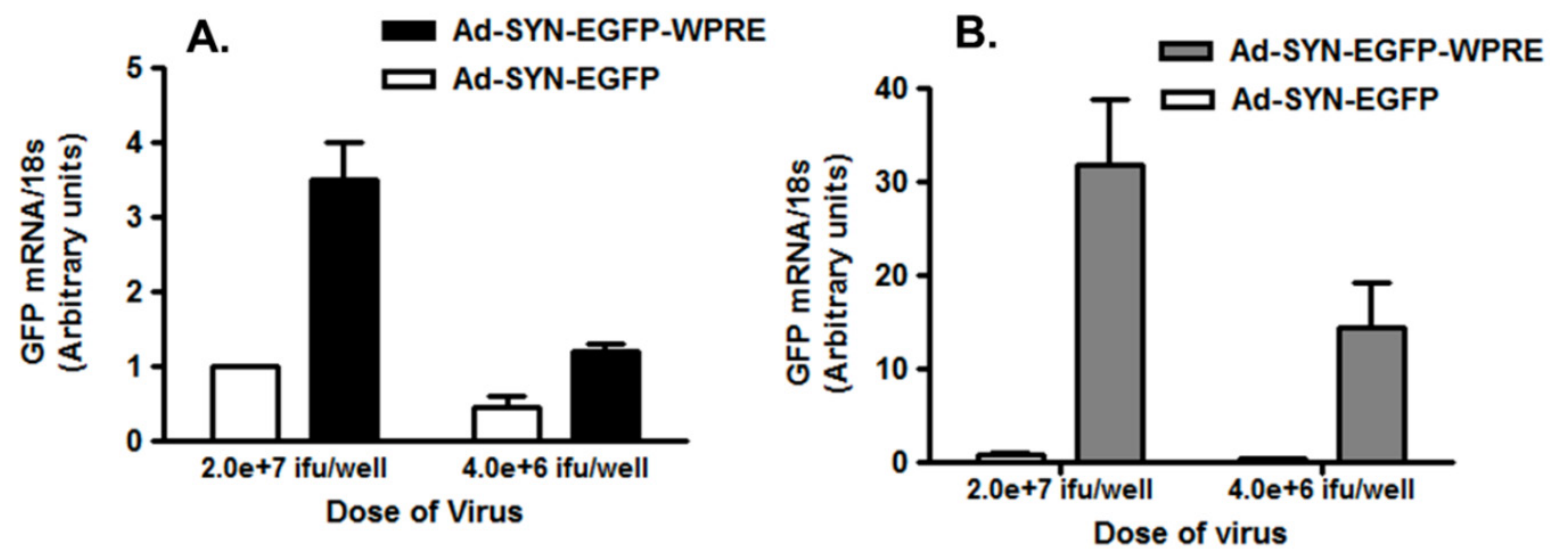

Figure 3. Expression of GFP in brain cell cultures transduced with Ad5-SYN- EGFP-WPRE or Ad5-SYN-EGFP. Primary cerebral cortical neurons (panel A) or astroglial cultures (panel B) were transduced with Ad5-SYN- EGFP-WPRE or Ad5-SYN-EGFP for 3 days as described in the methods. Levels of EGFP mRNA were analyzed via real time RT-PCR. Data are means \pm SEM from 3 experiments, are normalized to I8s RNA and are presented as arbitrary units. 


\section{Ad-SYN-EGFP-WPRE induced a high level of EGFP expression with no loss of neuronal specificity in the rat PVN}

To assess the effect of the WPRE on synapsin promoter activity in vivo, $5 \times 10^{6}$ ifu of Ad-SYN-EGFP-WPRE was microinjected into the PVN and EGFP levels were observed by fluorescence microscopy at one month post-injection (Figure 4). The results demonstrated that Ad-SYN-EGFP-WPRE mediated a long-term (at least 1 month), high level of EGFP expression, with no loss of neuronal specificity in the rat PVN based on overlap with NeuN immunoreactivity (Figure 4). This expression occurred despite the ability of this vector to elicit EGFP expression in astrocytes in vitro (Figure 3B).

The Ad-SYN-EGFP elicited very minor expression of GFP mRNA in astroglia as shown in Figure 3B. However, the expression of GFP mRNA was not significantly different between the two doses $\left(2.0 \times 10^{7}\right.$ ifu/well and 5.0x106 ifu/well) used. Moreover, based on our immunostaining data (not shown), we have never observed any glial expression of GFP protein using either the high dose or low dose of Ad-SYN-EGFP. These results may suggest that the astroglial GFP expression elicited by Ad-SYN-EGFP-WPRE is due to a direct influence of the WPRE on the SYN promoter.
rAAV2 elicits a high level of neuron specific over expression of foreign genes in cardiovascular control regions of the brain

Incubation of rat cortical neuronal cultures with AAV2-CBA-EGFP-WPRE (5 x10 viral gc/well) resulted in a high level of neuron-specific GFP expression, as shown by co-labeling with immunoreactive NeuN (Figure 2, panels J-L). In vivo, microinjection of AAV2-CBA-EGFP-WPRE $\left(1 \mu \mathrm{l}\right.$ of $\left.1 \times 10^{9} \mathrm{gc}\right)$ into the PVN, NTS or RVLM of adult SD rats elicited significant neuronal expression of EGFP two months after the injection. The fluorescence micrographs in Figure 5 demonstrate EGFP within neurons in the PVN (Fig.5a), NTS (Figure 5b) and RVLM (Figure 5c) at 2 months post injection, as evidenced by co-localization with the neuron specific marker NeuN. Finally, microinjection of AAV2-CBA-AT2R $\left(1 \mu \mathrm{l}\right.$ of $\left.5.0 \times 10^{8} \mathrm{gc}\right)$ into the RVLM of adult SD rats produced a high level of AT2 receptor expression within 7-14 days which was sustained to the end of the experiment (4.5 months) (Figure 6), as evidenced by receptor autoradiography. Microinjection of AAV2-CBA-EGFPWPRE into the RVLM of adult SD rats elicited no increases in AT2R at this site, as evidenced by autoradiography (data not shown).

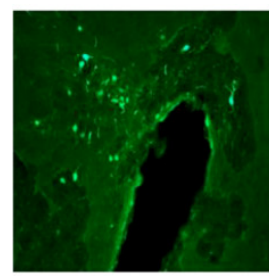

A

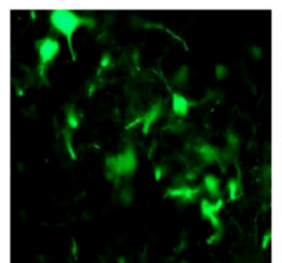

D

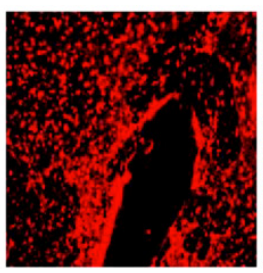

B

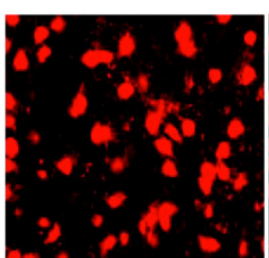

E
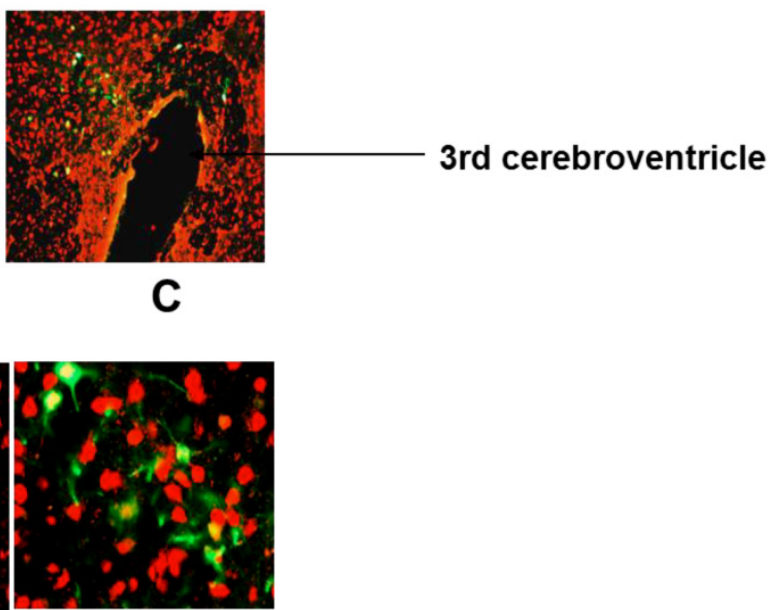

$\mathbf{F}$

Figure 4. Ad-SYN-EGFP-WPRE mediated transduction of EGFP into rat PVN. Ad-SYN-EGFP-WPRE $\left(I \mu l\right.$ of $5 \times 10^{6}$ ifu $)$ was microinjected into SD rat PVN, and I-month later brains were prepared for detection of EGFP fluorescence and immunostaining. A and $D$ are representative fluorescence micrographs showing EGFP in the PVN of an Ad-SYN-EGFP-WPRE injected rat; $B$ and E are NeuN immunostaining of the same area shown in $A$ and $D ; C$ and $F$ is the overlap between $A$ and $B$ or $D$ and $E$ respectively. $A-C, 20 x$ magnification; D-F, 400x magnification. 


\section{$5 \mathbf{a}$}
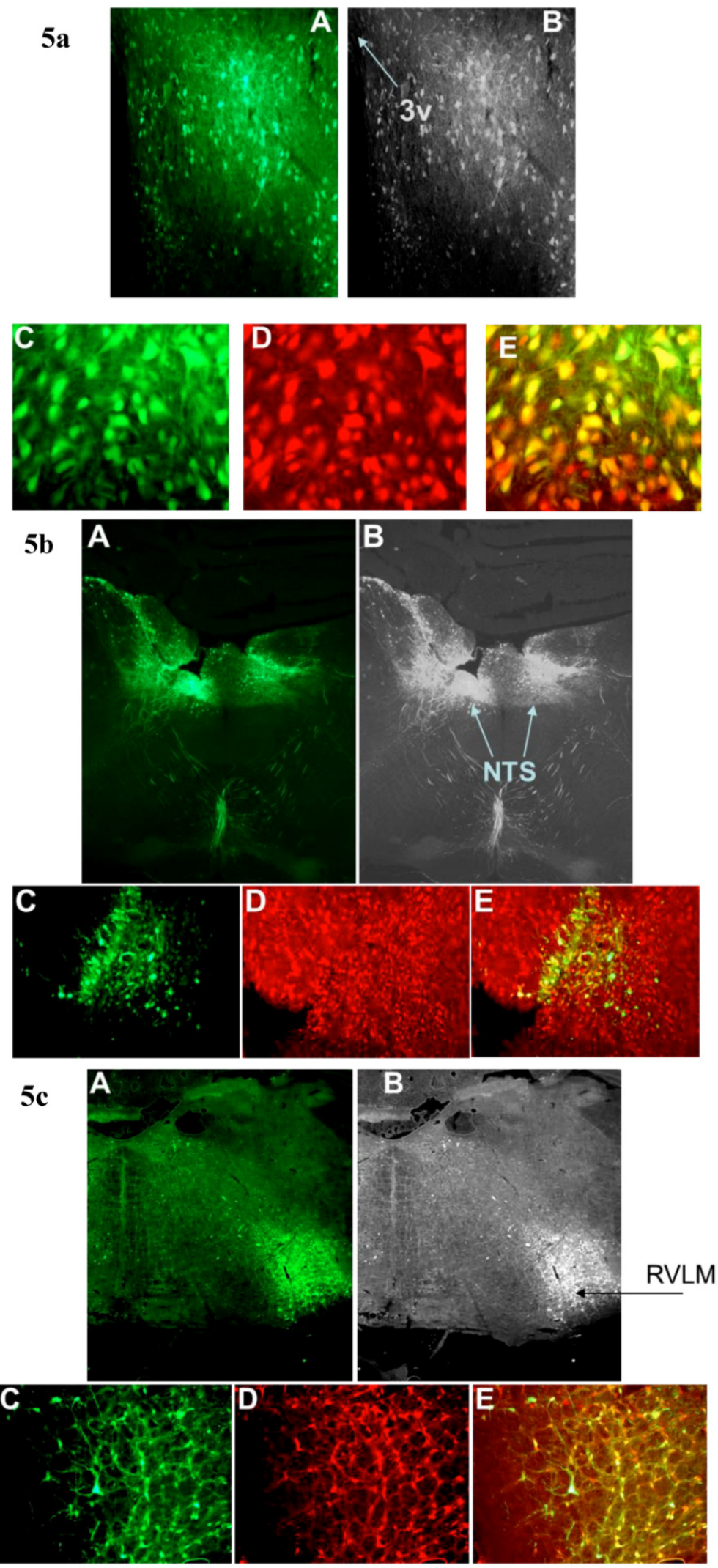

Figure 5. AAV2-CBA-EGFP-WPRE-mediated neuronal transduction of GFP into rat PVN, NTS and RVLM. (a) Panel (A) is a representative fluorescence micrograph (20x magnification) demonstrating the localization of GFP two months after injection of AAV2-CBA-EGFP-WPRE ( $1 \mu \mathrm{l}$ of $\left.1.0 \times 10^{\circ} \mathrm{gc}\right)$ into the PVN. Panel $(B)$ is a grayscale view of the picture from A, showing the location of the third cerebroventricle $(3 \mathrm{v})$. (C), (D) and $(E)$ are higher power fluorescence micrographs from the PVN (I00x magnification) showing respectively the presence of GFP, NeuN immunoreactivity and the overlap between the two. (b) and (c) Same as for (a), except that vector injections were made in the NTS and RVLM respectively. 


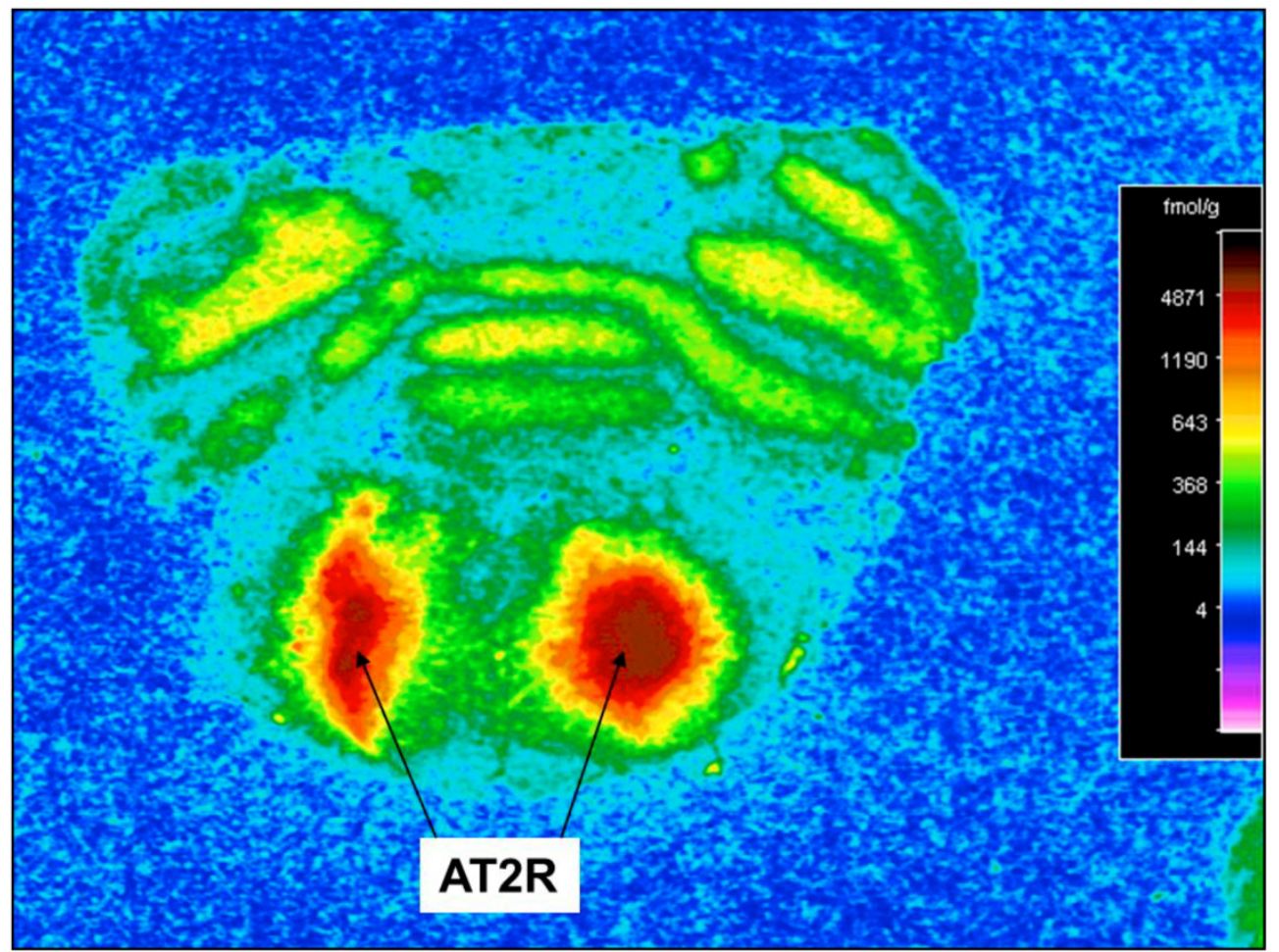

Figure 6. AAV2-CBA-AT2R-WPRE-mediated transduction of AT2R into SD rat RVLM. Receptor autoradiography was performed using Sar'-1le ${ }^{8}$-Ang II to determine the expression of AT2R in the RVLM of SD rats injected with AAV2-CBA-AT2R-WPRE (I $\mu \mathrm{l}$ of $\left.5.0 \times 10^{8} \mathrm{gc}\right)$. At 4.5 months after the injection, the rats were euthanized, brains were isolated, and receptor autoradiography was performed as described in the Methods. A representative autoradiogram is presented showing AT2R-specific binding in the region of the RVLM.

\section{Discussion}

Currently, perhaps the most popular approach for investigating gene function is to generate a transgenic or knockout animal in which the resulting phenotype may give clues. In fact, a vast number of transgenic or knockout mice are commercially available. However, the transgenic or knockout technology has a number of drawbacks which limit its value in physiological genomics, especially when it comes to understanding highly complicated systems such as central cardiovascular control networks. One particular problem of the transgenic or knockout strategy is a lack of spatial/cellular specificity and temporal control. Considering this problem, it is well known that central cardiovascular control is performed by a set of coordinated brain nuclei all of which perform different functions. Therefore, site-specific genetic perturbation is an important requirement for experiments addressing functional genomics of central blood pressure control[22].

To interpret the outcome of a genetic manipulation, at least in the context of central cardiovascular control, it is essential to restrict the modification to specific nuclei or, even better, to a specific cell type within that nucleus and to have temporal control over the expression of transgenes. One way to meet all of these requirements is to use somatic gene transfer directed towards selected nuclei in order to increase or decrease expression of a particular gene. Blood pressure can easily be measured allowing one to determine the effects of delivered transgenes in a prolonged experiment. However, transfer of genes into an intact brain in vivo remains a challenging task. While transfections of cell lines including those of neural origin are relatively trivial using various transfection reagents, mature neurons in the living brain are either resistant to these procedures or susceptible to damage. This is why viral vectors, which can effectively deliver transgenes into brain cells and even integrate them into the host genome for long-term expression, are so attractive.

In this study, we developed an adenoviral vector containing both the WPRE and the neuronal specific SYN promoter. The SYN promoter is reported to be neuron specific alone[16] or in conjunction with the WPRE[5]. However, the SYN1 promoter was also observed to be either a little leaky, at least within dissociated neuronal cultures, or less specific[2]. Our in vivo studies using a viral microinjection technique in rats demonstrated that 3 ' addition of the WPRE increased EGFP expression for at least one month with no loss of 
neuronal specificity in the PVN. It is also reported that a similar SYN-EGFP-WPRE cassette mediated long-term expression in rat hippocampus [5]. However, in vitro, the latter vector also produced much higher levels of transduction into glia in the presence of high levels of glial cells compared with the vector Ad-SYN-EGFP without WPRE. The mechanism whereby WPRE increased expression of foreign genes in glia is unclear. This may be due to differences between cell cultures, e.g. cell type and differentiation stage, and probably the presence of certain cellular factors such CRM-1, which has been shown to be at least partly required for WPRE activity[23]. The WPRE $(\approx 600 \mathrm{bp}$ in size) is a cis-acting tripartite structure derived from the woodchuck hepatitis virus (WHV) DNA genome, which acts post-transcriptionally to increase both nuclear and cytoplasmic unspliced RNA levels when inserted in the $3^{\prime}$ untranslated region[7]. It is thought that the WPRE acts early to increase transgene expression, possibly by directing post-transcriptional processing $[7,24]$. It has been found that WPRE-mediated enhancement of gene expression is promoter and cell line specific[25].

rAAV2 has been the most studied serotype in the brain and transduces exclusively neurons in the CNS[26]. Although rAAV1, rAAV5, rAAV8, rAAV9 and rAAV10 have been shown to transduce more neurons than rAAV2 injected at similar titers and with the same transcriptional cassette and produce a greater spread of foreign genes [27-29], rAAV2 is able to mediate significant expression of foreign genes in small cardiovascular control regions of the brain, such as the PVN, NTS and RVLM [30-32]. In addition, rAAV2 can be purified by using a simple single-step column purification based on its affinity to heparin, without need of a high level of manual and technical skills ( $\mathrm{CsCl} 2$ gradient centrifugation, iodixanol/heparin or HPLC purification). These indicate the advantages of rAAV2 for use in studies on central cardiovascular control mechanisms.

In summary, both rAAV2 and adenoviral vector are able to mediate specific neuronal overexpression of foreign genes in the cardiovascular control regions of rat brains, and the expression elicited by rAAV2 can last at least 4.5 months. The use of these vectors in future studies may allow us to uncover some of the physiological functions of certain genes in these regions.

\section{Acknowledgments}

We thank Dr. Sebastian Kügler (University Medicine Göttingen, Germany) for the human synapsin-1 promoter -containing plasmid. This work was supported by NIH Grant HL 076803 (C.S.), National Natural Science Foundation of China Grant 81072113 (H.L.) and National 863 High Technique Development Project of China Grant 2012AA02A403 (H.L.).

\section{Competing Interests}

The authors have declared that no competing interest exists.

\section{References}

1. Sinnayah P, Lindley TE, Staber PD, Cassell MD, Davidson BL, Davisson RL: Selective gene transfer to key cardiovascular regions of the brain: comparison of two viral vector systems. Hypertension 2002, 39(2 Pt 2):603-608.

2. Boulos S, Meloni BP, Arthur PG, Bojarski C, Knuckey NW: Assessment of CMV, RSV and SYN1 promoters and the woodchuck post-transcriptional regulatory element in adenovirus vectors for transgene expression in cortical neuronal cultures. Brain Res 2006, 1102(1):27-38.

3. Urban A, Rossier J. Genetic targeting of specific neuronal cell types in the cerebral cortex. Prog Brain Res. 2012;196:163-92.

4. Thomas CE, Schiedner G, Kochanek S, Castro MG, Lowenstein PR: Peripheral infection with adenovirus causes unexpected long-term brain inflammation in animals injected intracranially with first-generation, but not with high-capacity, adenovirus vectors: toward realistic long-term neurological gene therapy for chronic diseases. Proc Natl Acad Sci U S A 2000, 97(13):7482-7487.

5. Glover CP, Bienemann AS, Heywood DJ, Cosgrave AS, Uney JB: Adenoviral-mediated, high-level, cell-specific transgene expression: a SYN1-WPRE cassette mediates increased transgene expression with no loss of neuron specificity. Mol Ther 2002, 5(5 Pt 1):509-516.

6. Li H, Gao Y, Freire CD, Raizada MK, Toney GM, Sumners C: Macrophage migration inhibitory factor in the PVN attenuates the central pressor and dipsogenic actions of angiotensin II. Faseb J 2006, 20(10):1748-1750.

7. Donello JE, Loeb JE, Hope TJ: Woodchuck hepatitis virus contains a tripartite posttranscriptional regulatory element. J Virol 1998, 72(6):5085-5092.

8. Mandel RJ, Manfredsson FP, Foust KD, Rising A, Reimsnider S, Nash K, Burger C: Recombinant adeno-associated viral vectors as therapeutic agents to treat neurological disorders. Mol Ther 2006, 13(3):463-483.

9. Salegio EA, Samaranch L, et al. Safety study of adeno-associated virus serotype 2-mediated humanacid sphingomyelinase expression in the nonhuman primate brain. Hum Gene Ther.2012 Aug;23(8):891-902.

10. Betley JN, Sternson SM. Adeno-associated viral vectors for mapping, monitoring, and manipulating neural circuits. Hum Gene Ther. 2011;22(6):669-77.

11. Mancuso K, Hauswirth WW, Li Q, Connor TB, Kuchenbecker JA, Mauck MC, Neitz J, Neitz M. Gene therapy for red-green colour blindness in adult primates. ature.2009 Oct 8;461(7265):784-7.

12. Sun C, Li H, Leng L, Raizada MK, Bucala R, Sumners C: Macrophage migration inhibitory factor: an intracellular inhibitor of angiotensin II-induced increases in neuronal activity. J Neurosci 2004, 24(44):9944-9952.

13. Auricchio A, Hildinger M, O'Connor E, Gao GP, Wilson JM: Isolation of highly infectious and pure adeno-associated virus type 2 vectors with a single-step gravity-flow column. Hum Gene Ther 2001, 12(1):71-76.

14. Rohr UP, Wulf MA, Stahn S, Steidl U, Haas R, Kronenwett R: Fast and reliable titration of recombinant adeno-associated virus type- 2 using quantitative real-time PCR. J Virol Methods 2002, 106(1):81-88.

15. Sumners C, Tang W, Zelezna B, Raizada MK: Angiotensin II receptor subtypes are coupled with distinct signal-transduction mechanisms in neurons and astrocytes from rat brain. Proc Natl Acad Sci U S A 1991, 88(17):7567-7571.

16. Li HW, Gao YX, Raizada MK, Sumners C: Intronic enhancement of angiotensin II type 2 receptor transgene expression in vitro and in vivo. Biochem Biophys Res Commun 2005, 336(1):29-35.

17. Li HW, Gao YX, Matsuura T, Martynyuk A, Raizada MK, Sumners C: Adenoviral-mediated neuron specific transduction of angiotensin II type 2 receptors. Regul Pept 2005, 126(3):213-222.

18. Rowe BP, Saylor DL, Speth RC: Analysis of angiotensin II receptor subtypes in individual rat brain nuclei. Neuroendocrinology 1992, 55(5):563-573 
19. Daubert DL, Meadows GG, Wang JH, Sanchez PJ, Speth RC: Changes in angiotensin II receptors in dopamine-rich regions of the mouse brain with age and ethanol consumption. Brain Res 1999, 816(1):8-16.

20. Kugler S, Meyn L, Holzmuller H, Gerhardt E, Isenmann S, Schulz JB, Bahr M: Neuron-specific expression of therapeutic proteins: evaluation of different cellular promoters in recombinant adenoviral vectors. Mol Cell Neurosci 2001, 17(1):78-96.

21. Shaw G, Morse S, Ararat M, Graham FL: Preferential transformation of human neuronal cells by human adenoviruses and the origin of HEK 293 cells. Faseb J 2002, 16(8):869-871.

22. Kasparov S, Teschemacher AG, Hwang DY, Kim KS, Lonergan T, Paton JF: Viral vectors as tools for studies of central cardiovascular control. Prog Biophys Mol Biol 2004, 84(2-3):251-277.

23. Popa I, Harris ME, Donello JE, Hope TJ: CRM1-dependent function of a cis-acting RNA export element. Mol Cell Biol 2002, 22(7):2057-2067.

24. Zufferey R, Donello JE, Trono D, Hope TJ: Woodchuck hepatitis virus posttranscriptional regulatory element enhances expression of transgenes delivered by retroviral vectors. J Virol 1999, 73(4):2886-2892.

25. Klein R, Ruttkowski B, Knapp E, Salmons B, Gunzburg WH, Hohenadl C: WPRE-mediated enhancement of gene expression is promoter and cell line specific. Gene 2006, 372:153-161.

26. Burger C, Nash K, Mandel RJ: Recombinant adeno-associated viral vectors in the nervous system. Hum Gene Ther 2005, 16(7):781-791.

27. Burger C, Gorbatyuk OS, Velardo MJ, Peden CS, Williams P, Zolotukhin S, Reier PJ, Mandel RJ, Muzyczka N: Recombinant AAV viral vectors pseudotyped with viral capsids from serotypes 1, 2, and 5 display differential efficiency and cell tropism after delivery to different regions of the central nervous system. Mol Ther 2004, 10(2):302-317.

28. Reimsnider S, Manfredsson FP, Muzyczka N, Mandel RJ: Time course of transgene expression after intrastriatal pseudotyped rAAV2/1, rAAV2/2, rAAV2/5, and rAAV2/ 8 transduction in the rat. Mol Ther 2007, 15(8):1504-1511.

29. Sondhi D, Johnson L, Purpura K, Monette S, Souweidane MM, Kaplitt MG, Kosofsky B, Yohay K, Ballon D, Dyke J, Kaminksy SM, Hackett NR, Crystal RG. Long-Term Expression and Safety of Administration of AAVrh.10hCLN2 to the Brain of Rats and Nonhuman Primates for the Treatment of Late Infantile Neuronal Ceroid Lipofuscinosis. Hum Gene Ther Methods. 2012 23:324-35.

30. Freiria-Oliveira AH, Blanch GT, Li H, Colombari E, Colombari DS, Sumners C. Macrophage migration inhibitory factor in the nucleus of solitary tract decreases blood pressure in SHRs. Cardiovasc Res. 2013 97:153-60

31. Colombari E, Colombari DS, Li H, Shi P, Dong Y, Jiang N, Raizada MK, Sumners C, Murphy D, Paton JF. Macrophage migration inhibitory factor in the paraventricular nucleus plays a major role in the sympathoexcitatory response to salt. Hypertension. 2010 Nov;56(5):956-63.

32. Li H, Gao Y, Qi Y, Katovich MJ, Jiang N, Braseth LN, Scheuer DA, Shi P, Sumners C. Macrophage migration inhibitory factor in hypothalamic paraventricular nucleus neurons decreases blood pressure in spontaneously hypertensive rats. FASEB J. 2008 Sep;22(9):3175-85. 